\title{
Low Surface Brightness Galaxies in Deep Surveys
}

\author{
Henry. C. Ferguson \\ Space Telescope Science Institute, 3700 San Martin Drive, Baltimore \\ $M D$ 21218, USA
}

\begin{abstract}
.
We examine the constraints that can be placed on the space density of low-surface-brightness galaxies from deep HST images. Such images, while covering only a small solid angle, provide enough depth and spatial resolution to detect LSB galaxies at moderate redshift and distinguish them from galaxies of higher surface brightness.

We consider five simple models of the non-evolving or slowly-evolving population of LSB galaxies, motivated by various discussions in the recent literature. The basic results are (1) models with a large space-density of giant LSB galaxies at moderate redshift do not look like the real world and, (2) models with a large space-density of dwarf LSB galaxies are consistent with HST data (that is, they do not produce more faint LSB galaxies per unit solid angle than are detected at magnitudes $I \gtrsim 23$ ), but these LSB dwarf galaxies do not contribute much to faint galaxy counts unless they formed their stars in a rapid burst.
\end{abstract}

\section{Background}

The "faint blue galaxy problem" has garnered much attention over the last 10 years. The problem is basically that counts of galaxies rise more steeply toward faint magnitudes than expected from models with $\Omega_{\text {Matter }} \approx 1$. Reconciling the counts to $\Omega_{M}=1$ within the context of traditional "pure luminosity evolution" models for galaxies would require a fairly late $(z \leqslant 3)$ and bright formation epoch for galaxy spheroids (Yoshii and Takahara 1988). Such evolution should in principle show up in the redshift distribution, and was not observed. Only 5 years ago, when $\Omega_{M}=1$ was the preferred model, this discrepancy was considered something of a crisis. While solutions involving a cosmological constant were proposed (Yoshii and Peterson 1994; Fukugita et al. 1990), and worked reasonably well, solutions involving additional populations of galaxies were also considered. Among these hypothesised galaxy populations were LSB galaxies and dwarf galaxies. In the last few years the landscape has changed and models with low $\Omega$ and/or a cosmological constant no longer seem far-fetched. Large populations of LSB or dwarf galaxies may no longer be necessary to "solve" the faint blue galaxy problem. Nevertheless the physical motivations for postulating their existence are still valid. It is thus worth revisiting the issue to see if faint galaxy surveys themselves can provide limits on the allowable distribution of galaxy surface brightnesses and luminosity at low redshift. 
McGaugh (1994) was among the first to suggest that LSB galaxies could dominate faint galaxy counts. He pointed out that the isophotal detection limits of deep imaging surveys are far below those of the photographic surveys on which estimates of the local luminosity function were based. With isophotal detection thresholds of roughly $24.5 \mathrm{mag} \operatorname{arcsec}^{-2}$, the photographic surveys would miss even relatively bright LSB galaxies. Furthermore, the isophotal thresholds affect the photometry of galaxies near the detection limit, leading to systematic biases in estimates of the luminosity function. The detection thresholds of deep CCD surveys (e.g. Tyson 1988) are about three magnitudes fainter and could thus pick up LSB galaxies at low to moderate redshift. The blue colors and weak clustering of local LSB galaxies also seemed to make them attractive candidates for faint-blue galaxies. Ferguson and McGaugh (1995) and McLeod and Rieke (1995) considered more detailed models of LSB galaxies and concluded that while they could not explain the galaxy counts for $\Omega_{M}=1$, they could reduce the size of the discrepancy.

\section{Recent Developments}

The possibility that LSB galaxies could dominate the counts at $B \sim 25$ has been ruled out by improved angular-diameter measurements from the HST Medium Deep Survey and Hubble Deep Field projects (e.g. Roche et al. 1996; Williams et al. 1996). Typical half-light radii of galaxies at $B=25$ are about 0.3 arcsec, significantly smaller than predicted by the Ferguson and McGaugh (1995) model. The HDF size distribution and selection boundaries are described in more detail by Ferguson (1998).

Nevertheless, at roughly the same time as these new measurements have been ruling out large populations of LSB galaxies at faint magnitudes, deeper wide-area surveys have been improving estimates of the surface-brightness distribution of nearby galaxies, and confirming that it is broad (Bothun, Impey, and McGaugh 1997). Indeed, the number of galaxies per unit surface brightness seems to be nearly constant, or only slowly declining, to central surface brightnesses as faint as $\mu_{B}=24$. Of course, the surface brightness distribution is just one projection of the bivariate luminosity-surface-brightness distribution. It hard to tell from the exisiting data whether the LSB galaxies are mostly dwarf galaxies, or whether the SB distribution for giant galaxies is similarly broad. On the one hand the bivariate $\left(\mu_{0}, M\right)$ distribution derived by de Jong (1996) suggests that the distribution of $\mu_{0}$ at fixed absolute magnitude widens toward low luminosities, while on the other hand many of the LSB galaxies detected in the POSS-II survey (Schombert et al. 1992) turn out to have HI line widths larger than $100 \mathrm{~km} \mathrm{~s}^{-1}$.

The other projection of the bivariate brightness distribution, the luminosity function (LF), has also seen some recent developments (Marzke et al. 1998; Loveday 1997; Smith, Driver, and Phillipps 1997; Sawicki, Lin, and Yee 1997; Lin et al. 1996). In particular there appears to be an emerging consensus that the faint end slope of the LF is steep: $N(L) \propto L^{-\alpha}$ with $\alpha \lesssim-1.2$. Values as steep as $\alpha=-2.8$ have been reported for dwarf galaxies with $-12 \lesssim M_{B} \lesssim-16$ (Loveday 1997). The Hubble Deep Field (HDF), with a detection limit $V \approx 29$, can detect a flat-spectrum galaxy with $M_{B}=-14$ out to $z=0.7$ if it has 
sufficiently high surface brightness. Thus the HDF number counts can be used to test whether such estimates of the faint end slope make sense when coupled with a broad SB distribution.

Another development is the attempt to link the SB distribution to the angular momentum distribution of galaxies predicted from tidal torquing in the early universe (Dalcanton, Spergel, and Summers 1997; Mo, Mao, and White 1998). The Dalcanton et al. (1997) model starts with a log-normal distribution of spin angular momenta. The dark matter is assumed to collapse initially into a halo with a Hernquist density profile, which is later modified in response to the cooling and condensation of the baryons into a disk near the center of the potential. This $a b$ initio prediction of the bivariate brightness distribution provides a physically motivated hypothesis that can now be tested against the observations.

\section{Five Easy Models}

In the remainder of this presentation, I will explore the implications of these recent $\mathrm{SB}$ and $\mathrm{LF}$ distributions for the predicted properties of galaxies in the HDF. The purpose here is not to try to reproduce the galaxy counts, but instead to see if any of the recently inferred distribution functions Bothun, Impey, and McGaugh (1997, Dalcanton, Spergel, and Summers (1997) overpredict the counts of LSB galaxies in the HDF.

The modeling procedure, which involves Monte-Carlo sampling of the assumed bivariate brightness distribution, is described in detail by Ferguson and McGaugh (1995) and Ferguson and Babul (1998). For all models, the assumed cosmology has $H_{0}=65 \mathrm{~km} \mathrm{~s}^{-1} \mathrm{Mpc}^{-1}, \Omega_{M}=0.1$ and $\Omega_{\Lambda}=0$. The luminosity function has the Schechter (1976) form

$$
\phi(L) d L=\phi^{*}\left(L / L^{*}\right)^{\alpha} e^{-L / L^{*}} d\left(L / L^{*}\right) .
$$

The LF normalization, $\phi^{*}=2.8 \times 10^{-2} h_{65}^{3} \mathrm{Mpc}^{-3}$, is set to correspond to half the total galaxy population for the luminosity function normalization recommended by Ellis (1997). The luminosity function runs from $0.003 L^{*}$ to $10 L^{*}$ for all models. For the non-evolving models, the galaxies are given spectral energy distributions that correspond to a model with a star-formation e-folding time $\tau=10^{10} \mathrm{yr}$ and a metallicity 0.05 solar. This model matches the typical colors for LSB galaxies locally. Galaxies are distributed with constant space density, and, for models 1-3 and 5, are assumed not to evolve.

\section{Model 1}

The first simulation is a non-evolving model using the McGaugh (1996) SB distribution function:

$$
\log \phi\left(\mu_{0}\right)=m\left(\mu_{0}-\mu_{0}^{*}\right)
$$

with

$$
\begin{gathered}
m=-0.3 \text { for } \mu_{0}>\mu_{0} * \\
m=2.6 \text { for } \mu_{0}<\mu_{0} *
\end{gathered}
$$



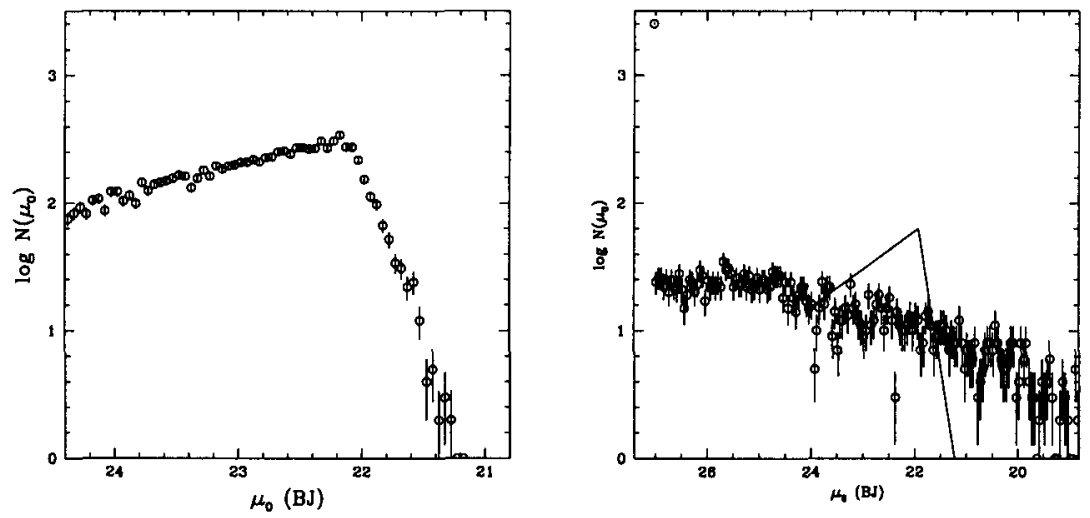

Figure 1. SB distributions for models 1-4 (Left), and model 5 (right). The SB distribution in the left panel is reproduced as thin lines in the right panel. The "data" points are galaxies drawn from Monte-Carlo realizations of the analytic distribution functions.

and

$$
\mu_{0} *=21.9 B_{J} \text { at } z=0 .
$$

The SB distribution is shown in Fig. 1. The luminosity function (Fig. 2 ) is given a steep slope $\alpha=-1.8$, and a characteristic absolute magnitude $M_{B J}^{*}=-20.5+5 \log h_{65}^{2}$. The model dramatically overpredicts the number of LSB in the HDF (Fig. 3). This is not surprising given that this model is also strongly inconsistent with the Loveday et al. (1992) luminosity function.

\section{Model 2}

The second simulation is the same as the first, but the LF is assumed to have a faint-end slope $\alpha=-1$, consistent with Loveday et al. (1992). While such a model falls far short of matching galxy counts at HDF depths, nevertheless, it predicts more large LSB galaxies than are seen. Specifically, the model predicts that there should be about 12 galaxies in the HDF with isophotal areas (above the detection threshold) of more than 1.6 square arcsec, with a mean $I$-band surface brightness within the central 0.2 " fainter than $\mu_{0, I}=24.5$. The actual HDF has only two galaxies meeting these criteria. The likely explanation for the discrepancy is that surface brightness is correlated with luminosity, and that the bulk of the LSB galaxies are dwarfs.

\section{Model 3}

The third simulation adopts the same $\alpha=-1.8$ slope as model 1 , but uses a characteristic absolute magnitude $M_{B J}^{*}=-16$. Thus in this model, the LSB galaxies are almost all dwarfs. The inputs and results are not shown in the figures as they are nearly identical to those of model 4 . 

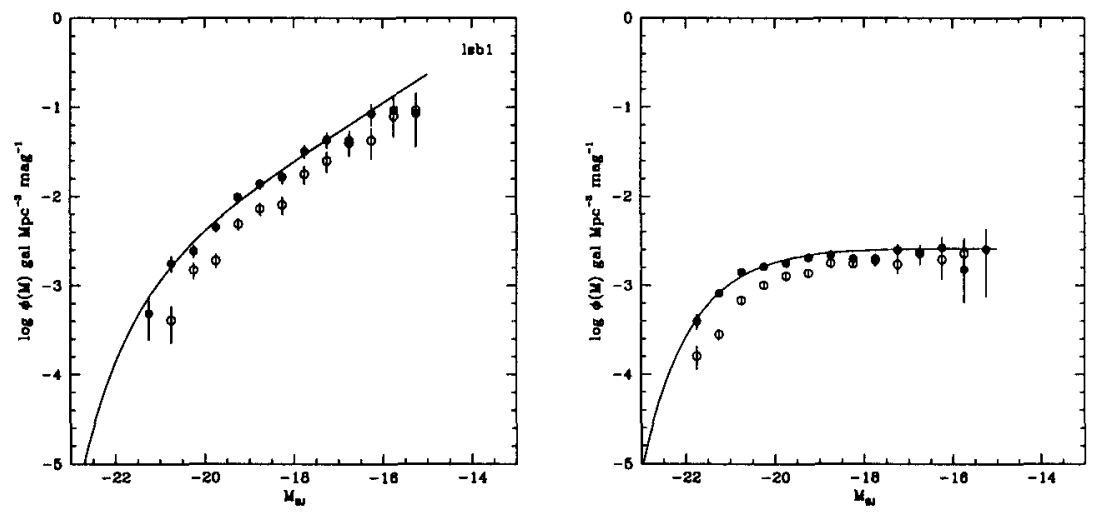

Figure 2. Luminosity functions at $z=0$ for 1 and 2. The solid curve is the adopted model. The black dots are the luminosity function in a Monte-Carlo realization of the model not accounting for selection biases. The open circles are the luminosity function that would be recovered in a survey with the selection function of the Loveday et al. (1992) survey.
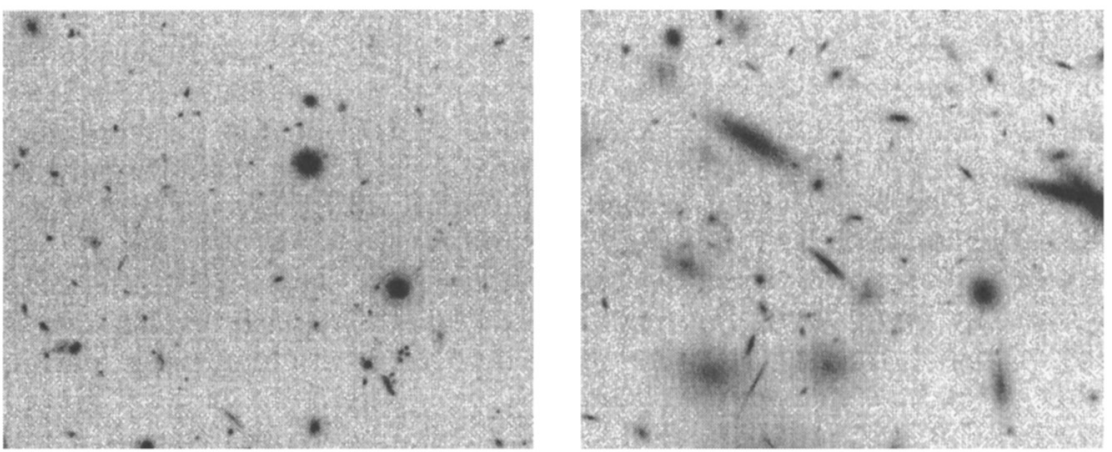

Figure 3. Left: A portion of the HDF image (WF chip 4). Right: simulated portion of the HDF image from model 1. 

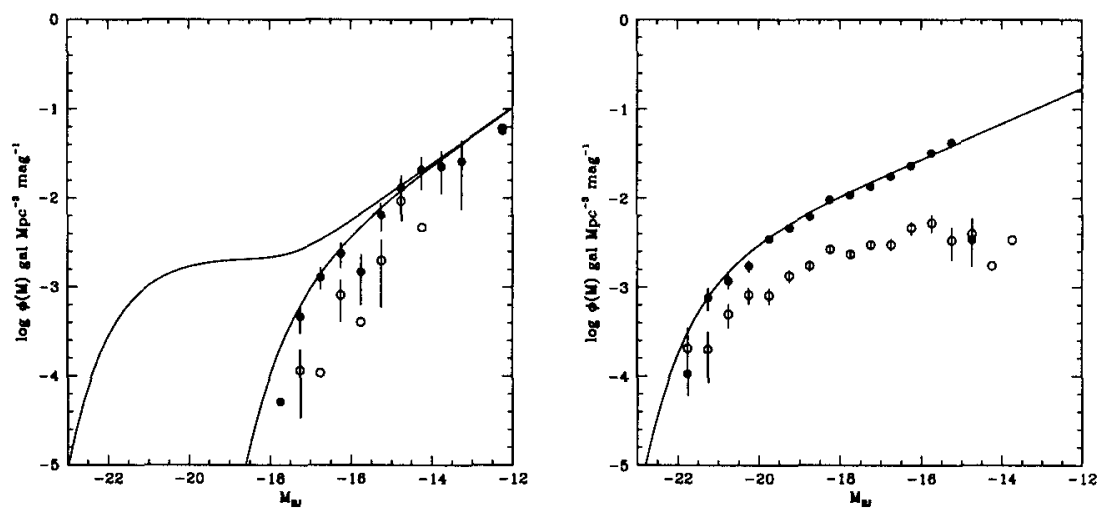

Figure 4. Luminosity functions at $z=0$ for models 4 and 5. Symbols have the same meaning as Fig. 2.

\section{Model 4}

This model is the same as model 3 , but the stellar populations are allowed to evolve. Galaxies are assumed to form at redshifts between $z=1$ and $z=$ 2.5 , with a constant rate of formation in this interval. The spectral energy distributions evolve according to the $\tau=10 \mathrm{Gyr}$ low-metallicity model used for the non-evolving models. The luminosity function for this model is shown in Fig. 4 and the simulated HDF image in Fig. 5. Models 3 and 4 do not overpredict the number of LSB galaxies in the HDF. That is, a large population of LSB dwarfs could exist out to arbitrary redshift and not violate the constraints of the HDF.

On the other hand, the models show that dwarf galaxies drawn from the McGaugh (1996) SB distribution contribute almost negligibly to the deep counts, even with a steep $L F$ slope $\alpha=-1.8$. The galaxies in this model make up less than $10 \%$ of the galaxy population fainter than $V_{606}=23$.

\section{Model 5}

Model 5 uses the Dalcanton, Spergel, and Summers (1997) SB distribution, which depends on the baryon fraction $F$, the specific angular momentum $\lambda$, the logarithmic spread in specific angular momentum $\sigma_{\lambda}$, and several other parameters. To first order, the model gives a central surface brightness $\Sigma_{0} \propto$ $F M_{\text {tot }}^{1 / 3} \lambda^{-(2+6 F)}$. We have taken $F=0.05,\langle\lambda\rangle=0.06$ and $\sigma_{\lambda}=0.7$, and adjusted the other parameters so that the peak of the SB distribution at fixed scale length $r_{0}=2 \mathrm{kpc}$ is $\mu_{0}=24$. The SB distribution predicted by this model (Fig. 1) is broader than the empirical one of McGaugh (1996), but in this case there is some dependence on luminosity. The luminosity function slope is taken to be $\alpha=-1.5$ with $M_{B J}^{*}=-20.5$. The luminosity function for this model is shown in Fig. 4. 

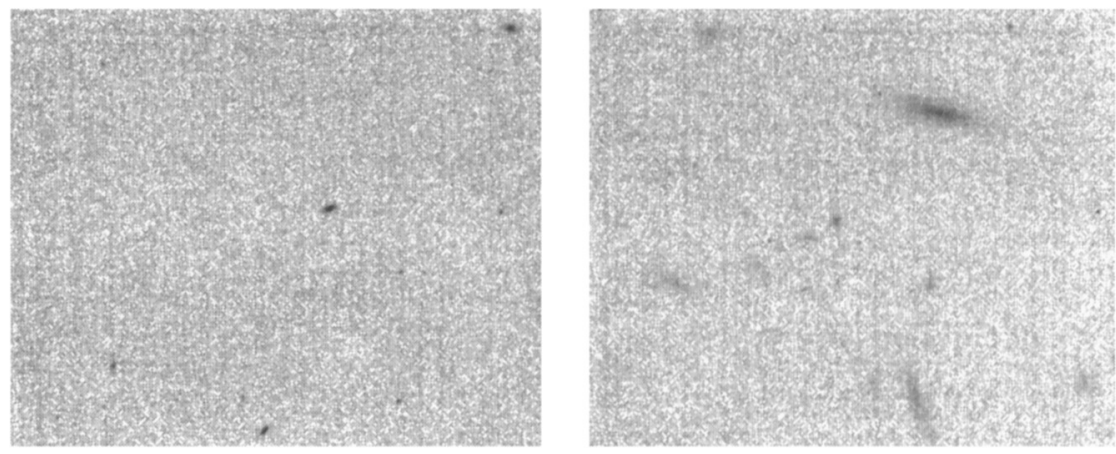

Figure 5. Left: Model 4. Right: Model 5.

As was the case for models 1 and 2, this model overpredicts the number of large-angular-size LSB galaxies in the HDF (Fig. 5).

\section{Summary}

Galaxy counts for the different models (determined by running FOCAS on the simulated images) are shown in Table 1 . Model 1 is the only one that predicts LSB galaxies are significant contributors to the counts, but it shows the clearest disagreement with the observed HDF size distribution. Models 2 and 5 are less strongly ruled out, and models 3 and 4 are consistent with the data. But in these cases the LSB galaxy population makes up at most about $20 \%$ of the counts fainter than $V_{606}=23$.

Table 1. Galaxy Counts for the different Models.

\begin{tabular}{clllll}
$V_{606}$ & HDF & Model 1 & Model 2 & Model 4 & Model 5 \\
\hline $23-25$ & 128 & 126 & 10 & 4 & 24 \\
$25-27$ & 466 & 404 & 40 & 25 & 108 \\
$27-29$ & 1078 & 915 & 106 & 118 & 209
\end{tabular}

The surface brightness distributions considered here are relatively "flat," with nearly constant numbers of galaxies per logarithmic interval of surface brightness. The simulations indicate that flat distributions with many of $g i$ ant LSB galaxies produce too many faint LSB galaxies to be consistent with the HDF. The discrepancy could be avoided if giant LSB galaxies formed only recently (e.g., at $z \lesssim 0.3$ ).

Flat SB distributions with only dwarfs are consistent with the $\mathrm{HDF}$, but a slowly evolving popuation of LSB dwarfs probably contributes less than $20 \%$ of the faint-galaxy counts for any plausible luminosity function. 


\section{References}

Bothun, G., Impey, C., and McGaugh, S. S. 1997, PASP, 109, 745

Dalcanton, J. J., Spergel, D. N., and Summers, F. J. 1997, ApJ, 482, 659

de Jong, R. S. 1996, A\&A, 313, 45

Ellis, R. S. 1997, ARA\&A, 35, 389

Ferguson, H. C. 1998, in The Hubble Deep Field, ed. M. Livio, S. M. Fall, and P. Madau (Cambridge, Cambridge University Press), p. 181

Ferguson, H. C. and Babul, A. 1998, MNRAS, in press

Ferguson, H. C. and McGaugh, S. S. 1995, ApJ, 440, 470

Fukugita, M., Yamashita, K., Takahara, F., and Yoshii, Y. 1990, ApJ, 361, L1

Lin, H., Kirshner, R. P., Schectman, S. A., Landy, S. D., Oemler, A., Tucker, D. L., and Schechter, P. L. 1996, ApJ, 464, 60

Loveday, J. 1997, ApJ, 489, 29

Loveday, J., Peterson, B. A., Efstathiou, G., and Maddox, S. 1992, ApJ, 390, 338

Marzke, R. O., Da Costa, L. N., Pellegrini, P. S., Willmer, C. N. A., and Geller, M. J. 1998, ApJ, 503, 617

McGaugh, S. 1996, MNRAS, 280, 337

McGaugh, S. S. 1994, Nature, 367, 538

McLeod, B. A. and Rieke, M. J. 1995, ApJ, 454, 611

Mo, H. J., Mao, S., and White, S. D. M. 1998, MNRAS, 295, 319

Roche, N., Ratnatunga, K., Griffiths, R. E., Im, M., and Neuschaefer, L. 1996, MNRAS, 282, 1247

Sawicki, M. J., Lin, H., and Yee, H. K. C. 1997, AJ, 113, 1

Schechter, P. 1976, ApJ, 203, 297

Schombert, J. M., Bothun, G. D., Schneider, S. E., and McGaugh, S. S. 1992, AJ, 103, 1107

Smith, R., Driver, S. P., and Phillipps, S. 1997, MNRAS, 287, 415

Tyson, J. A. 1988, AJ, 96, 1

Williams, R. E. et al. 1996, AJ, 112, 1335

Yoshii, Y. and Peterson, B. 1994, ApJ, 436, 551

Yoshii, Y. and Takahara, F. 1988, ApJ, 326, 1 